\title{
A decade of healthcare improvement in cystic fibrosis: lessons for other chronic diseases
}

\section{David P Stevens, ${ }^{1}$ Bruce C Marshall ${ }^{2}$}

${ }^{1}$ The Dartmouth Institute for Health Policy and Clinical Practice, Lebanon, New Hampshire, USA

${ }^{2}$ The Cystic Fibrosis Foundation, Bethesda, Maryland, USA

\section{Correspondence to} David P Stevens, The Dartmouth Institute for Health Policy and Clinical Practice, 30 Lafayette Street, Lebanon, NH 03756 , USA;

david.p.stevens@dartmouth.edu

Received 25 January 2014 Accepted 27 January 2014
CrossMark

To cite: Stevens DP, Marshall BC. BMJ Qual Saf 2014;23:i1-i2.
From 2002 to 2012, the median predicted survival age for people with cystic fibrosis (CF) increased nearly 10 yearsfrom 31.3 years to 41.1 years. ${ }^{1}{ }^{2}$ Strategic efforts to improve care for CF marked this remarkable era. These efforts were supported by expert leadership among CF healthcare improvement professionals and resources from the CF Foundation. While process outcomes improved substantially-for example, more timely clinic visits, increased influenza immunisation rates, and more effective screening for associated problems such as depression and diabetes, ${ }^{1}{ }^{2}$ clinical outcomes improved even more dramaticallynotably, pulmonary function, nutritional status and predicted survival.

This supplement, Ten Years of Improvement Innovation in Cystic Fibrosis Care, captures the larger perspective of this comprehensive improvement initiative and reports representative CF care centre-level examples. It also identifies strategies to widen the circle of improvement professionals who successfully publish their innovative work in scholarly journals. ${ }^{3}$

\section{WHAT CAN BE FOUND IN THIS SUPPLEMENT?}

Institution-based improvement reports in this supplement describe better airway clearance linked to patient education, ${ }^{4}$ successful strategies to meet guidelines for regular CF clinic visits, ${ }^{5}$ local care improvement to meet and sustain goals for nutritional status, ${ }^{6}$ reduced airway infection rates through improved infection prevention and control measures, ${ }^{7}$ implementation of care redesign to improve the management of acute exacerbations of $\mathrm{CF}^{8}$ and an initiative to facilitate the continuum of care for patients moving from paediatric to internal medicine specialists, important as CF increasingly becomes a disease of both children and adults. ${ }^{9}$
Additional commissioned papers describe overarching improvement approaches that include strategic benchmarking, ${ }^{10}$ development of a transparent patient registry to track outcomes at the individual patient and CF care centre (system) levels, ${ }^{11}$ effective integration of patients and families into improvement processes, ${ }^{12} 13$ and a series of improvement collaboratives to spread knowledge for improvement among CF care centres. ${ }^{1} 14$

\section{WHAT WORKED TO IMPROVE CF CARE?}

Disaggregating the relative contributions of these various improvement strategies is difficult. ${ }^{15}$ Schechter et al make a strong case for the influence of a patient registry, ${ }^{11}$ while Boyle et al craft a compelling argument for the role played by benchmarking - the analysis and spread of improvement strategies from highly achieving institutions to the wider CF centre network. ${ }^{10}$ In addition, there is face value for the strong role that patients and families perform in improving CF care. ${ }^{12} 13$ Finally, participation by care centre personnel in improvement collaboratives has been associated with demonstrably improved process outcomes. $^{14}$

Recent reviews have provided empiric evidence for the impact of selected elements of context in effective improvement initiatives $^{16}{ }^{17}$-for example, leadership, culture and information systems. In their critical review, Kaplan et a ${ }^{17}$ found a particularly positive role for institutional and microsystem quality improvement team leadership, with the strongest case being made for active participation by senior leaders in improvement initiatives, and the effective communication by leaders of their vision and expectations.

We argue for the strong contribution provided by effective integrated leadership from the CF Foundation as well as national 
leaders represented by many of the authors in this supplement. Other contributing contextual elements ${ }^{16} 17$ in the CF improvement story include effective data management, substantial physician involvement, a pervasive culture of improvement among CF care centres, and microsystem-level motivation for change.

\section{CONCLUSIONS}

While we do not underestimate the impact of recent scientific and therapeutic advances in $\mathrm{CF},{ }^{1}$ we argue that healthcare improvement strategies such as those reported here-tested and adopted in CF care centres over a decade-have had a profound role in the improvement of life and predicted survival for people with CF. Moreover, we suggest that the aggregate effects of these combined efforts-a widely available and transparent patient registry, benchmarking of best improvement practices, active involvement of patients and their families in improvement initiatives, and dissemination of improvement methodologies among CF healthcare professionals-may be transferrable to improving care for others with serious chronic illnesses.

Contributors Both authors contributed to the design of this initiative. Both contributed to the original and subsequent drafts of this report.

Competing interests DPS received honoraria and travel support from the Cystic Fibrosis Foundation for preparation of this supplement. He is a member of the SQUIRE Development Group for which he receives honoraria and travel support from The Health Foundation and the Robert Wood Johnson Foundation. BCM receives support for salary and professional expenses from the Cystic Fibrosis Foundation.

Provenance and peer review Commissioned; internally peer reviewed.

\section{REFERENCES}

1 Marshall BC, Nelson E. Accelerating implementation of biomedical research advances: critical elements of a successful 10-year CF Foundation healthcare delivery improvement initiative. BMJ Qual Saf 2014;23:i95-103.

2 Mogayzel P, Dunitz J, Marrow L, et al. Improving chronic care delivery and outcomes: the impact of the Cystic Fibrosis Care Center Network. BMJ Qual Saf 2014;23:i3-i8.

3 Stevens DP, Marshall BC. Healthcare improvement is incomplete until it is published: the cystic fibrosis initiative to support scholarly publication. BMJ Qual Saf 2014;23: i104-107.
4 Zanni R, Sembrano E, Du D, et al. The impact of re-education of airway clearance techniques (REACT) on adherence and pulmonary function in patients with cystic fibrosis. BMJ Qual Saf 2014;23:i50-55.

5 Berlnski A, Chambers MJ, Willis L, et al. Redesigning care to meet national recommendation of 4 or more yearly clinic visits in patients with cystic fibrosis. BMJ Qual Saf 2014;23:i42-49.

6 Savant A, Britton L, Petren K, et al. Sustained improvement in nutritional outcomes at two pediatric cystic fibrosis centers after quality improvement collaboratives. BMJ Qual Saf 2014;23: i81-89.

7 Savant A, O’Malley C, Bichl S, et al. Improved patient safety through reduced airway infection rates in a pediatric cystic fibrosis program after a quality improvement effort to enhance infection prevention and control measures. BMJ Qual Saf 2014;23:173-80.

8 Antos N, Quintero D, Walsh-Kelly C, et al. Improving cystic fibrosis exacerbation care-two success stories. BMJ Qual Saf 2014;23:i33-41.

9 Okumura M, Ong D, Dawson D, et al. Improving transition from pediatric to adult cystic fibrosis care: program implementation and evaluation. BMJ Qual Saf 2014;23:i64-72.

10 Boyle M, Sabadosa K, Quinton H, et al. Key findings of the Cystic Fibrosis Foundation's US Clinical Practice Benchmarking Project. BMJ Qual Saf 2014;23:115-22.

11 Schechter M, Fink A, Homa K, et al. The Cystic Fibrosis Foundation patient registry as a tool for use in quality improvement. BMJ Qual Saf 2014;23:19-14.

12 Sabadosa K, Batalden P. The interdependent roles of patients. families, and professionals: a system for the co-production of healthcare and its improvement. BMJ Qual Saf 2014;23: i90-94.

13 Siracusa C, Weiland J, Acton J, et al. The impact of transforming healthcare delivery on cystic fibrosis outcomes: a decade of quality improvement at Cincinnati Children's Hospital. BMJ Qual Saf 2014;23:i56-63.

14 Godfrey MM, Oliver BJ. Accelerating improvement in cystic fibrosis care: the learning and leadership collaborative model. BMJ Qual Saf 2014;23:i23-32.

15 Dixon-Woods M, Bosk CL, Aveling E-L, et al. Explaining Michigan: developing an ex post theory of a quality improvement program. Milbank Quarterly 2011;89: 167-205.

16 Taylor SL, Dy S, Foy R, et al. What context features might be important determinants of the effectiveness of patient safety interventions? BMJ Qual Saf 2011;20:611-7.

17 Kaplan HC, Brady PW, Dunitz MC, et al. The influence of context on improvement success in health care: a systematic review of the literature. Milbank Quarterly 2010;88: 500-59. 